\title{
Article \\ Is Chronic Varicocele a Risk Factor for Secondary Hyperparathyroidism?
}

\author{
Rossella Cannarella ${ }^{1}$ (D), Rosita A. Condorelli ${ }^{1}(\mathbb{D})$, Sarah Perelli ${ }^{1}$, Aldo E. Calogero ${ }^{1}\left(\mathbb{D}\right.$, Emanuela Greco $^{2}$, \\ Antonio Aversa ${ }^{3}$ (D) and Sandro La Vignera ${ }^{1, *(\mathbb{D})}$
}

1 Department of Clinical and Experimental Medicine, University of Catania, 95123 Catania, Italy rossella.cannarella@phd.unict.it (R.C.); rosita.condorelli@unict.it (R.A.C.); sarah.perelli@libero.it (S.P.); acaloger@unict.it (A.E.C.)

2 Department of Health Sciences, University “Magna Graecia”, 88100 Catanzaro, Italy; emanuela.greco@unicz.it

3 Department of Experimental and Clinical Medicine, University "Magna Graecia", 88100 Catanzaro, Italy; aversa@unicz.it

* Correspondence: sandrolavignera@unict.it; Fax: +39-95-3781180

check for updates

Citation: Cannarella, R.; Condorelli, R.A.; Perelli, S.; Calogero, A.E.; Greco, E.; Aversa, A.; La Vignera, S. Is Chronic Varicocele a Risk Factor for Secondary Hyperparathyroidism? J Clin. Med. 2022, 11, 716. https:// doi.org/10.3390/jcm11030716

Academic Editor: Du Geon Moon

Received: 17 November 2021

Accepted: 27 January 2022

Published: 28 January 2022

Publisher's Note: MDPI stays neutral with regard to jurisdictional claims in published maps and institutional affiliations.

Copyright: (C) 2022 by the authors. Licensee MDPI, Basel, Switzerland. This article is an open access article distributed under the terms and conditions of the Creative Commons Attribution (CC BY) license (https:// creativecommons.org/licenses/by/ $4.0 /$ )

\begin{abstract}
Objective: To assess whether varicocele affects testicular 25-hydroxylase activity. Methods: Twenty normozoospermic patients with bilateral varicocele (grade III according to the Dubin and Amelar classification) without indications to undergo varicocele repair (normal sperm parameters and testicular volume; no scrotal pain) were consecutively enrolled and followed-up for four years. Serum levels of parathyroid hormone (PTH), calcium, and 25-hydroxy-cholecalciferol [25(OH)D] along with serum luteinizing hormone ( $\mathrm{LH})$, follicle-stimulating hormone (FSH), total testosterone (TT), conventional sperm parameters, sperm DNA fragmentation (SDF) rate, and testicular volume (TV) were measured annually for three years. PTH, calcium, and $25(\mathrm{OH}) \mathrm{D}$ serum levels over time were compared with those of age- and body mass index (BMI)-matched control group of twenty varicocelectomized patients. Main results: Both intra- and between-group analyses showed that serum PTH levels increased significantly over time in parallel with a significant decline in $25(\mathrm{OH}) \mathrm{D}$ levels. Serum calcium levels did not change significantly. At the same time, signs of mild Leydig and Sertoli cell dysfunction were found, such as an increase in gonadotropins and decreased TT and VT. However, conventional sperm parameters and SDF rate did not change significantly. Conclusion: This prospective controlled study provides the first evidence of a negative impact of bilateral grade III varicocele on testicular 25-hydroxylase activity. Accordingly, the patients included in this study showed a significant increase in PTH and a decrease in 25(OH)D levels over time. Patients with varicocele deserve endocrinologic counseling.
\end{abstract}

Keywords: 25-hydroxylase; varicocele; vitamin D; parathyroid hormone; testicular function

\section{Introduction}

Testicular varicocele is an abnormal dilation and tortuosity of the pampiniform plexus that drains the testis. Its prevalence has been estimated to be up to $15 \%$ in adulthood. It is higher in infertile patients who have a prevalence of $19-41 \%$ in primary infertility and up to $80 \%$ in secondary infertility [1]. Clinical staging of varicocele severity can be done using the Dubin and Amelar classification. It consists of four grades: grade 0 which identifies the subclinical varicocele (not clinically detectable but diagnosed by ultrasound); grade I, palpable only during the Valsalva maneuver; grade II, appreciable even without Valsalva maneuver; grade III, visible on inspection [2].

Several lines of evidence show a negative role of varicocele on testicular function. Indeed, patients with varicocele have lower sperm quality and pregnancy outcomes compared to healthy controls [3-5]. Furthermore, meta-analytic studies have shown its negative influence on Leydig cell function and, therefore, on testosterone biosynthesis [6]. 
The exact mechanisms by which varicocele damage the testicular function are not entirely clear. Several theories have been proposed. First of all, varicocele causes scrotal hyperthermia that negatively impacts spermatogenesis. Furthermore, a decreased heat-shock protein (HSP) expression may contribute to heat stress that, in turn, increases oxidative stress (OS) and apoptosis. Moreover, blood stasis in varicose veins can promote the entrapment of leucocytes that release reactive oxygen species (ROS). Finally, varicocele can cause testicular hypoxia. Reduced expression of anti-apoptotic genes and increased expression of pro-apoptotic genes increases the susceptibility to OS and thermogenic damage. The different degrees of susceptibility may explain why varicocele, even of a severe degree, is not always associated with testicular damage [7].

So far, the effects of varicocele on testicular 25-hydroxylase activity have not been evaluated. This microsomal enzyme, codified by the CYP2R1 gene, has been identified in human Leydig cells [8]. It catalyzes the hydroxylation of cholecalciferol into 25-hydroxycholecalciferol [25(OH)D]. This is further hydroxylated into 1,25( $\left.\mathrm{OH}_{2}\right)$-vitamin D3 (calcitriol) in the kidney under the stimulation of parathyroid hormone (PTH). Calcitriol, the active form of vitamin $\mathrm{D}$, stimulates the absorption of calcium from the kidney tubules and bowel and favors bone mineralization. Several other genomic and non-genomic effects of calcitriol have been reported in many tissues [9].

In healthy testis, the expression of 25-hydroxylase is higher than that reported in the liver. However, its expression is reduced in testis with hypospermatogenesis or Sertoli cell-only syndrome (SCOS), and this predisposes to $25(\mathrm{OH}) \mathrm{D}$ deficiency [8]. Furthermore, patients with late-onset hypogonadism are at risk of developing 25(OH)D deficiency and secondary hyperparathyroidism. This has been shown by studies investigating the best therapeutic scheme for Vitamin D replacement, able to normalize both $25(\mathrm{OH}) \mathrm{D}$ and serum parathyroid hormone (PTH) levels in these patients [10]. These findings suggest that patients with diseases that lead to testicular dysfunction should not only undergo clinical management for abnormalities of sperm parameters or decreased testosterone biosynthesis but also serum $25(\mathrm{OH}) \mathrm{D}$ levels resulting from Leydig cell dysfunction.

Whether varicocele can affect the activity of 25-hydroxylase in Leydig cells is not known. Therefore, this study aimed to evaluate this topic. To accomplish this, twenty consecutive normozoospermic patients with bilateral varicocele of grade III (according to the Dubin and Amelar scale) were prospectively evaluated for PTH, calcium, and 25(OH)D (primary outcomes) for three years. PTH, calcium, and $25(\mathrm{OH}) \mathrm{D}$ serum levels over time were compared with those of age- and body mass index (BMI)-matched control group of twenty varicocelectomized patients. The secondary outcomes were serum gonadotropin and total testosterone (TT) levels, conventional sperm parameters, sperm DNA fragmentation (SDF) rate, and testicular volume (TV).

\section{Subjects and Methods}

\subsection{Patient Selection}

This is a prospective study performed in men who referred to the Division of Endocrinology, Metabolic Diseases and Nutrition, University of Catania, for varicocele, from March 2020. We selected the first 20 patients with specific clinical features. The inclusion criteria were: bilateral varicocele of grade III according to the Dubin and Amelar scale [2] and without the following indications to varicocele repair: normal sperm parameters, no scrotal pain, and TV in the normal range $(>15 \mathrm{~mL})$ by scrotal ultrasound. Furthermore, the selected patients did not have a head injury, endocrine disorders (hypogonadism, hyperprolactinemia, Cushing syndrome, acromegaly, hypopituitarism), elevated serum follicle-stimulating hormone (FSH) levels, systemic diseases (kidney and/or liver diseases), and genetic disorders. Finally, past history of hyperparathyroidism, hypercalcemia, and vitamin D supplementation at enrolment or during the study were considered exclusion criteria. Forty Caucasian patients were enrolled. Twenty did not undergo varicocele repair (Patient group) and twenty underwent varicocele repair (Control group). The patient group had a mean $( \pm S D)$ age of $29.9 \pm 6.2$ years, whereas the Control group had a mean 
$( \pm \mathrm{SD})$ age of $31.0 \pm 4.0$ years $(p>0.1)$. The two groups were also BMI-matched (Patients: $22.6 \pm 1.4 \mathrm{~kg} / \mathrm{m}^{2}$; Controls: $\left.23.5 \pm 2.0 \mathrm{~kg} / \mathrm{m}^{2} ; p>0.1\right)$. Each endpoint was measured at enrollment (T0) and annually for three years (T1, T2, and T3). This consisted of PTH, calcium, and $25(\mathrm{OH}) \mathrm{D}$ serum levels as primary outcomes. Serum luteinizing hormone (LH), FSH, and TT levels, conventional sperm parameters, SDF rate, and TV were evaluated as secondary outcomes.

\subsection{Hormonal Measurements}

Blood testing was performed in fasting conditions, at 8:00-9:00 am. The serum levels of LH, FSH, TT, PTH, calcium, and $25(\mathrm{OH}) \mathrm{D}$ were measured by electrochemiluminescence (Hitachi-Roche equipment, Cobas 6000, Roche Diagnostics, Indianapolis, IN, USA). The reference values were as follows: LH 1.14-8.75 IU/L, FSH 0.95-11.95 IU/L, TT 0.478-9.8 ng/mL, PTH 0.02-1.23 ng/mL, calcium 8.8-10.6 mg/dL, 25(OH)D 1-100 ng/mL.

\subsection{Sperm Analysis}

Semen samples were collected by masturbation into a sterile container after 2-7 days of sexual abstinence and were analyzed immediately after liquefaction. The analysis was conducted according to the $2010 \mathrm{WHO}$ guidelines. Each sample was evaluated for seminal volume, $\mathrm{pH}$, sperm count, progressive motility, morphology, and round cell concentration [11].

\subsection{Sperm DNA Fragmentation}

SDF was measured through flow cytometry by terminal deoxynucleotidyl transferasemediated deoxyuridine triphosphate nick-end labeling (TUNEL) staining, using an EPICS XL (Becker Coulter, Milan, Italy). The negative control was obtained by not adding terminal deoxynucleotidyl transferase to the reaction mix, while the positive control was obtained by pretreating spermatozoa with $1 \mathrm{mg} / \mathrm{mL}$ of RNase-free deoxyribonuclease I (Sigma Chemical, St. Louis, MO, USA) at $37^{\circ} \mathrm{C}$ for $60 \mathrm{~min}$ before labeling.

\subsection{Scrotal Ultrasound Evaluation}

TV was evaluated by scrotal ultrasound. The ultrasound examination was performed with a GX MegasEsaote (EsaoteSpA, Genoa, Italy) device, equipped with linear, highresolution, and high-frequency $(7.5$ to $14 \mathrm{MHz})$ probes dedicated to the study of soft body areas, with color Doppler for detecting slow flow and a scanning surface of at least $5 \mathrm{~cm}$. The TV was calculated using the ellipsoid formula (length $\times$ width $\times$ thickness $\times 0.52$ ). The testis was considered normal in size when it had a volume between 15 and $25 \mathrm{~cm}^{3}$, low normal when it had a volume between 10 and $12 \mathrm{~cm}^{3}$, and hypotrophic when it had a volume lower than $10 \mathrm{~cm}^{3}$ [12,13]. TV was calculated as the mean value between the volumes of the right and left testes.

\subsection{Statistical Analysis}

Results are reported as mean \pm SD throughout the study. The normal distribution of each variable was evaluated by using the Shapiro-Wilks test. Within-group differences were analyzed by one-way analysis of variance (ANOVA) followed by the Tukey-Kramer post hoc test. Statistical analysis was performed using MedCalc Software Ltd. (Ostend, Belgium) (Version 19.6-64 bit). A $p$ value lower than 0.05 was considered statistically significant.

\subsection{Ethical Approval}

This study was conducted at the Division of Endocrinology, Metabolic Diseases and Nutrition of the University teaching hospital "G. Rodolico-San Marco", University of Catania (Catania, Italy). The protocol was approved by the internal Institutional Review Board and informed written consent was obtained from each participant after a full explanation of the purpose and nature of all procedures used. The study has been conducted according to the principles expressed in the Declaration of Helsinki. 


\section{Results}

The overtime anthropometric, hormonal values, testicular volume, conventional sperm parameters, and SDF rate in the 20 patients with non-operated varicocele are shown in Table 1. A significant increase of gonadotropins and a decrease of TT and TV were observed, indicating a negative, although subclinical, the impact of varicocele on both Sertoli and Leydig cell function over time. In particular, serum FSH levels were significantly higher at $\mathrm{T} 2$ and $\mathrm{T} 3$ compared to T0, and serum LH levels were higher at all the follow-up time-points compared to T0. Furthermore, LH at T2 was higher than T1, and, at T3, it was higher than both $\mathrm{T} 1$ and $\mathrm{T} 2$, showing a time-dependent increase. TT was significantly lower at all the time points compared with T0. TV was lower at T2 and T3 compared with T0, and at T3 compared with T1. Finally, conventional sperm parameters and SDF rate did not show any significant change compared with T0 (Table 1).

Table 1. Trend over time of anthropometric, hormonal values, testicular volume, sperm parameters, and sperm DNA fragmentation in patients with III degrees bilateral varicocele.

\begin{tabular}{|c|c|c|c|c|}
\hline Parameters & $\begin{array}{c}\text { T0 } \\
(\text { Mean } \pm \text { SD) }\end{array}$ & $\begin{array}{c}\text { T1 } \\
(\text { Mean } \pm \text { SD) }\end{array}$ & $\begin{array}{c}\text { T2 } \\
(\text { Mean } \pm \text { SD) }\end{array}$ & $\begin{array}{c}\text { T3 } \\
(\text { Mean } \pm \mathrm{SD})\end{array}$ \\
\hline BMI $\left(\mathrm{Kg} / \mathrm{m}^{2}\right)$ & $22.6 \pm 1.4$ & $22.9 \pm 1.0$ & $23.0 \pm 0.8$ & $22.8 \pm 0.6$ \\
\hline FSH (IU/L) & $2.6 \pm 0.4$ & $3.0 \pm 0.6$ & $3.1 \pm 0.5^{*}$ & $3.2 \pm 0.5^{*}$ \\
\hline $\mathrm{LH}(\mathrm{IU} / \mathrm{L})$ & $2.3 \pm 0.5$ & $4.1 \pm 0.7 *$ & $5.5 \pm 1.4^{*, \dagger}$ & $6.6 \pm 1.5 * t, \ddagger$ \\
\hline $\mathrm{TT}(\mathrm{ng} / \mathrm{mL})$ & $5.6 \pm 0.4$ & $4.9 \pm 0.6^{*}$ & $4.7 \pm 0.6^{*}$ & $4.5 \pm 0.6^{*}$ \\
\hline Total TV (mL) & $16.8 \pm 1.3$ & $15.8 \pm 1.6$ & $15.1 \pm 1.7^{*}$ & $14.2 \pm 1.7^{*,+}$ \\
\hline Sperm concentration (million/mL) & $78.3 \pm 10.4$ & $71.8 \pm 8.9$ & $78.5 \pm 7.8$ & $71.1 \pm 9.5$ \\
\hline Sperm progressive motility (\%) & $51.1 \pm 7.1$ & $46.6 \pm 5.9$ & $47.4 \pm 6.1$ & $51.2 \pm 5.2$ \\
\hline $\begin{array}{l}\text { Spermatozoa with normal } \\
\text { morphology }(\%)\end{array}$ & $13.0 \pm 2.2$ & $12.0 \pm 1.5$ & $11.4 \pm 2.8$ & $12.6 \pm 1.7$ \\
\hline SDF rate $(\%)$ & $2.1 \pm 2.5$ & $1.8 \pm 0.9$ & $1.7 \pm 0.9$ & $1.9 \pm 0.9$ \\
\hline
\end{tabular}

Abbreviations: $\mathrm{BMI}=$ body mass index; $\mathrm{FSH}=$ follicle-stimulating hormone; $\mathrm{LH}=$ luteinizing hormone $\mathrm{SD}=$ standard deviation; $\mathrm{SDF}=$ sperm DNA fragmentation; $\mathrm{T} 0$ = baseline; $\mathrm{T} 1$ = 1-year follow-up; $\mathrm{T} 2$ = 2-year follow-up; T3 $=3$-year follow-up; TT $=$ total testosterone; TV $=$ testicular volume. ${ }^{*} p<0.05 \mathrm{vs.} \mathrm{T0;}{ }^{\dagger} p<0.05 \mathrm{vs}$ $\mathrm{T} 1 ; \ddagger p<0.05$ vs. T2. Within-group differences were analyzed by one-way analysis of variance (ANOVA) followed by the Tukey-Kramer post hoc test.

Interestingly, serum PTH levels showed an increasing trend over time and its serum levels increased compared to baseline at all the time points assessed in the Patient group. Furthermore, both the values at T2 and T3 were significantly higher than those at T1. These values were also significantly higher at $\mathrm{T} 1, \mathrm{~T} 2$, and $\mathrm{T} 3$ compared to the Control group (Figure 1A). Serum levels of calcium were significantly higher in T3 vs. T0 in the patient group. Compared to controls, patients showed significantly higher calcium serum levels at each assessed time. Additionally, patients and controls did not show serum calcium levels falling within the range of hypercalcemia (Figure 1B). Serum 25(OH)D showed decreasing trend over time in the Patient group. In particular, its levels decreased at all the time points assessed compared with T0. Additionally, 25(OH)D values at T2 and T3 were significantly lower than values at T1. Patients also showed significantly lower 25(OH)D serum levels at each assessed time compared to the control group (Figure 1C). Since the BMI of the enrolled patients did not change over time (Table 1), the decline of serum $25(\mathrm{OH}) \mathrm{D}$ levels does not seem related to an increase in the fat mass. These results indicate that the patients included in this study were at risk for developing serum $25(\mathrm{OH}) \mathrm{D}$ deficiency and secondary hyperparathyroidism, possibly due to a reduction of the activity of the testicular 25-hydroxylase. 

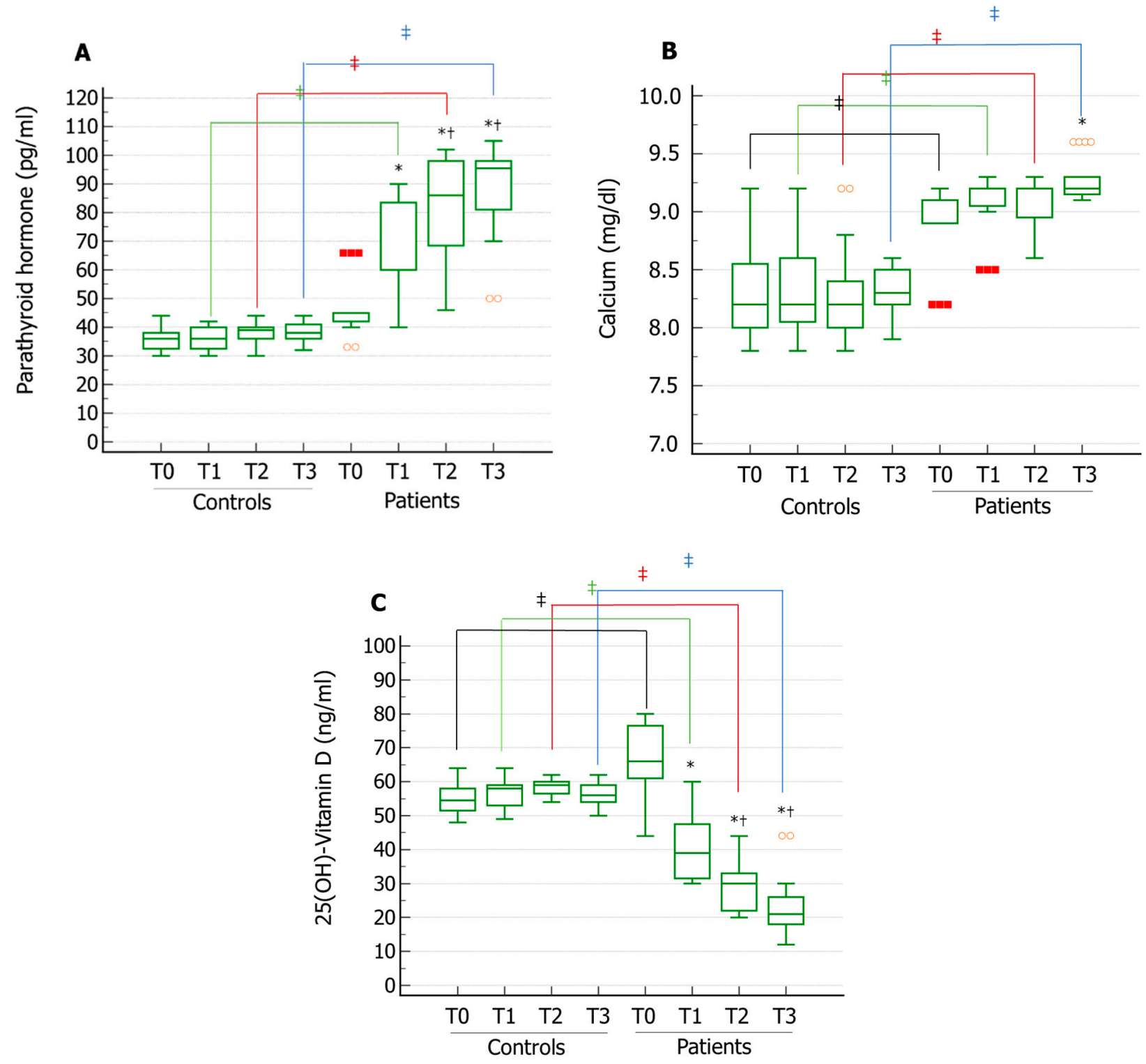

Figure 1. Serum levels of parathyroid hormone (PTH), calcium, and 25(OH)-Vitamin D in patients with III degree bilateral varicocele and varicocelectomized controls. Serum PTH levels significantly increased after one (T1), two (T2), and three (T3) years of follow-up compared to the values at enrolment (T0) in the patient group. Compared to controls, patients showed significantly higher PTH serum levels at T1, T2, and T3. (A). Serum calcium levels were significantly higher in T3 vs. T0 in the patient group. Compared to controls, patients showed significantly higher calcium serum levels at each assessed time. (B). Serum 25(OH)-Vitamin D levels significantly decreased over time in the patient group. Compared to controls, patients showed significantly lower 25(OH)-Vitamin D serum levels at each assessed time. (Panel (C)). ${ }^{*} p<0.05$ vs. ${ }^{1} 0 ;{ }^{\dagger} p<0.05$ vs. T1; ${ }^{\ddagger} p<0.05$.

\section{Discussion}

Varicocele is diagnosed in $15 \%$ of men [1] and, according to its severity and the patients susceptibility, it can lead to subclinical or clinical dysfunction of both Sertoli and Leydig cells. This can result in impaired conventional sperm parameters and pregnancy outcomes, increased gonadotropin levels, low serum TT levels, and even reduced TV.

Scanty evidence is currently available on the effect of varicocele on testicular CYP2R1 enzyme, which hydroxylases vitamin D into 25(OH)D. To evaluate this issue, we enrolled 20 consecutive patients with III degrees bilateral varicocele (according to the Dubin and Amelar classification), normal sperm parameter, and no indication of varicocele repair (no 
infertility, no scrotal pain, no decrease of testicular volume). They underwent measurements of serum PTH, calcium, and 25(OH)D at enrollment and annually for three years. Serum gonadotropin and TT levels, conventional sperm parameters, SDF rate, and TV were also evaluated. A significant increase of PTH and a specular decrease in 25(OH)D levels was observed over time. Concomitantly, signs of subclinical Leydig and Sertoli cell dysfunction, such as an increase in gonadotropins and a decrease in TT and TV over time was found. No change in conventional sperm parameters and SDF rate was observed.

These results suggest a decline of testicular 25-hydroxylase activity in these patients that explains the decrease of serum 25(OH)D levels and the consequent secondary hyperparathyroidism. This interpretation is further supported by the increase in serum LH levels. Accordingly, the testicular CYP2R1 activity has been reported to be LH dependent [14]. Furthermore, the administration with human chorionic gonadotropin (hCG) which has an LH-like activity, is indeed able to increase serum $25(\mathrm{OH}) \mathrm{D}$ levels in patients with lateonset hypogonadism and hypovitaminosis D [14]. Therefore, the increase in LH likely indicates Leydig cell dysfunction that concerns not only its steroidogenic activity but also that of 25-hydroxylase.

The decline of $25(\mathrm{OH}) \mathrm{D}$ that we observed in these patients does not seem related to changes in body weight. Indeed, vitamin D, due to its lipophilic nature, is stored in the adipose tissue, and obese patients have a greater chance of hypovitaminosis D [9]. It is likely that the reduction in vitamin $\mathrm{D}$ levels over time cannot be ascribed to a change in body composition in the enrolled patients, since the BMI does not change and that the patients included were of normal weight.

The activity of testicular steroidogenic enzymes can be influenced by thermic damage caused by varicocele. In adult rats with experimentally induced varicocele, the activities of the 17 $\alpha$-hydroxylase, 17,20-desmolase, and 17ß-hydroxysteroid dehydrogenase enzymes (involved all in testosterone biosynthesis) decrease significantly [15]. Likewise, a negative impact of varicocele also on the activity of the 25-hydroxylase may be hypothesized.

The results of the present study suggest, for the first time, that the management of bilateral, grade III, varicocele should include not only fertility and TT production but also the evaluation of PTH and Vitamin D. The long-term complications of bilateral varicocele may include, therefore, hypovitaminosis D and secondary hyperparathyroidism that, in turn, can affect bone mineralization, also in patients with no evidence of overt hypogonadism. However, there are no studies, so far, on the bone mineral density in patients with bilateral varicocele. In the next future, if further studies will confirm these results, indications for varicocele repair could include the onset of hypovitaminosis D and secondary hyperparathyroidism.

The strengths of this study include the prospective 3-year-long study design, the homogeneity of the cohort, the stringent inclusion criteria, and the presence of a control group. The main limitations of the study, in our opinion, are represented by lack of information regarding lower varicocele grades and inability to quantify the patients' exposure time to varicocele before enrollment.

In conclusion, this prospective study provides the first evidence of a negative impact of bilateral grade III varicocele on the testicular 25-hydroxylase activity. Accordingly, the patients included in this study showed a significant increase in serum PTH and a parallel decrease in $25(\mathrm{OH}) \mathrm{D}$ levels over time. If further studies will confirm this evidence, endocrinologic counseling may be included in the management of varicocele.

Author Contributions: S.L.V. and R.C. selected the subjects, critically analyzed the data, drafted and wrote the manuscript. S.L.V. and R.A.C. performed the clinical analysis, contributed to interpretation of data and to the writing of manuscript. A.E.C. and R.A.C. drafted and supervised the project and revised the manuscript critically. S.P., E.G. and A.A. validated the data. All authors have read and agreed to the published version of the manuscript.

Funding: This research did not receive any specific grant from funding agencies in the public, commercial, or not-for-profit sectors. 
Institutional Review Board Statement: The study was conducted in accordance with the Declaration of Helsinki, and approved by the internal Institutional Review Board, University teaching hospital “G. Rodolico-San Marco", University of Catania (42/2021).

Informed Consent Statement: Informed consent was obtained from all subjects involved in the study.

Data Availability Statement: Data are available upon request to the corresponding author.

Conflicts of Interest: The authors declare that there is no conflict of interest that could be perceived as prejudicing the impartiality of the research reported.

\section{References}

1. Agarwal, A.; Deepinder, F.; Cocuzza, M.; Agarwal, R.; Short, R.A.; Sabanegh, E.; Marmar, J.L. Efficacy of varicocelectomy in improving semen parameters: New meta-analytical approach. Urology 2007, 70, 532-538. [CrossRef]

2. Dubin, L.; Amelar, R.D. Varicocele size and results of varicocelectomy in selected subfertile men with varicocele. Fertil. Steril. 1970, 21, 606-609. [CrossRef]

3. La Vignera, S.; Condorelli, R.; Vicari, E.; D'Agata, R.; Calogero, A.E. Effects of varicocelectomy on sperm DNA fragmentation, mitochondrial function, chromatin condensation, and apoptosis. J. Androl. 2012, 33, 389-396. [CrossRef] [PubMed]

4. Kirby, E.W.; Wiener, L.E.; Rajanahally, S.; Crowell, K.; Coward, R.M. Undergoing varicocele repair before assisted reproduction improves pregnancy rate and live birth rate in azoospermic and oligospermic men with a varicocele: A systematic review and meta-analysis. Fertil. Steril. 2016, 106, 1338-1343. [CrossRef] [PubMed]

5. Mongioì, L.M.; Mammino, L.; Compagnone, M.; Condorelli, R.A.; Basile, A.; Alamo, A.; La Vignera, S.; Morgia, G.; Russo, G.I.; Calogero, A.E. Effects of Varicocele Treatment on Sperm Conventional Parameters: Surgical Varicocelectomy Versus Sclerotherapy. Cardiovasc. Intervent. Radiol. 2019, 42, 396-404. [CrossRef] [PubMed]

6. $\quad$ Li, F.; Yue, H.; Yamaguchi, K.; Okada, K.; Matsushita, K.; Ando, M.; Chiba, K.; Fujisawa, M. Effect of surgical repair on testosterone production in infertile men with varicocele: A meta-analysis. Int. J. Urol. 2012, 19, 149-154. [CrossRef] [PubMed]

7. Hassanin, A.M.; Ahmed, H.H.; Kaddah, A.N. A global view of the pathophysiology of varicocele. Andrology 2018, 6, 654-661. [CrossRef] [PubMed]

8. Foresta, C.; Strapazzon, G.; De Toni, L.; Perilli, L.; Di Mambro, A.; Muciaccia, B.; Sartori, L.; Selice, R. Bone mineral density and testicular failure: Evidence for a role of vitamin D 25-hydroxylase in human testis. J. Clin. Endocrinol. Metab. 2011, 96, E646-E652. [CrossRef] [PubMed]

9. Holick, M.F.; Binkley, N.C.; Bischoff-Ferrari, H.A.; Gordon, C.M.; Hanley, D.A.; Heaney, R.P.; Murad, M.H.; Weaver, C.M. Endocrine Society. Evaluation, treatment, and prevention of vitamin D deficiency: An Endocrine Society clinical practice guideline. J. Clin. Endocrinol. Metab. 2011, 96, 1911-1930, Erratum in J. Clin. Endocrinol. Metab. 2011, 96, 3908. PMID:21646368. [CrossRef] [PubMed]

10. Foresta, C.; Calogero, A.E.; Lombardo, F.; Lenzi, A.; Ferlin, A. Late-onset hypogonadism: Beyond testosterone. Asian J. Androl. 2015, 17, 236-238. [CrossRef] [PubMed]

11. World Health Organization. WHO Laboratory Manual for the Examination and Processing of Human Semen, 5th ed.; Cambridge University Press: Cambridge, UK, 2010.

12. Sakamoto, H.; Yajima, T.; Nagata, M.; Okumura, T.; Suzuki, K.; Ogawa, Y. Relationship between testicular size by ultrasonography and testicular function: Measurement of testicular length, width, and depth in patients with infertility. Int. J. Urol. 2008, 15, 529-533. [CrossRef] [PubMed]

13. Pilatz, A.; Rusz, A.; Wagenlehner, F.; Weidner, W.; Altinkilic, B. Reference values for testicular volume, epididymal head size and peak systolic velocity of the testicular artery in adult males measured by ultrasonography. Ultraschall Med. 2013, 34, 349-354. [CrossRef] [PubMed]

14. La Vignera, S.; Condorelli, R.A.; Cimino, L.; Russo, G.I.; Morgia, G.; Calogero, A.E. Late-onset hypogonadism: The advantages of treatment with human chorionic gonadotropin rather than testosterone. Aging Male 2016, 19, 34-39. [CrossRef] [PubMed]

15. Rajfer, J.; Turner, T.T.; Rivera, F.; Howards, S.S.; Sikka, S.C. Inhibition of testicular testosterone biosynthesis following experimental varicocele in rats. Biol. Reprod. 1987, 36, 933-937. [CrossRef] [PubMed] 